\title{
A Gravity Balancing Passive Exoskeleton for the Human Leg
}

\author{
Sunil K. Agrawal*, PhD, Sai K. Banala, Abbas Fattah, PhD \\ Mechanical Systems Laboratory, Department of Mechanical Engineering \\ *Professor and Corresponding author Email: agrawal@me.udel.edu,Tel: (302)831-8049 \\ John P. Scholz, PhD, Vijaya Krishnamoorthy, PhD, Wei-Li Hsu \\ Department of Physical Therapy
}

University of Delaware, Newark, DE 19716

\begin{abstract}
A gravity balancing lower extremity exoskeleton is a simple mechanical device composed of rigid links, joints and springs, which is adjustable to the geometry and inertia of the leg of a human subject wearing it. This passive exoskeleton does not use any motors or controllers, yet can still unload the human leg joints of the gravity load over the full range of motion of the leg. The underlying principle of gravity balancing consists of two steps: (i) Locate the combined system center of mass of the human leg and the exoskeleton, (ii) Add springs to the exoskeleton, one between the center of mass of the combined system and the fixed frame representing the trunk, the others within the links of the exoskeleton so that the potential energy of the combined system is invariant with configuration of the leg. Additionally, parameters of the exoskeleton can be changed to achieve a prescribed level of partial balancing, between 0-gravity and 1-gravity.

The goals of this paper are as follows: (i) briefly review the theory for gravity balancing and present laboratory prototypes of gravity balanced machines, (ii) describe the design of a lower extremity exoskeleton that was fabricated using this principle, and (iii) show the performance of the exoskeleton on both healthy human subjects and a stroke patient by comparison of leg muscle EMG recordings, joint range of motion, and measured joint torques. These results strongly suggest that human joints can be unloaded from gravity using these exoskeletons and the human joint range of motion can be significantly increased. Potential applications of gravity balancing exoskeletons include: (i) gait training of stroke patients, (ii) characterization of long-term effects of zero gravity on the human musculature, (iii) human performance augmentation during assembly tasks.
\end{abstract}

\section{INTRODUCTION}

Gravity plays an important role in human movement. An elderly person may have difficulty getting up from a chair as the musculature may not be strong enough to sustain the gravity loads at the joints during the movement. A person with a weak musculature or poor neuro-motor control may find it hard to swing a leg against gravity or walk but may find it easier to adapt and learn if the gravity was taken away from the joints. We believe that lower or upper extremity exoskeletons, that unload the human joints from gravity, can significantly enhance the human understanding of the role that gravity plays in human movement and can provide new insights into movement training. Additionally, the flexibility to prescribe partial gravity at the joints (between 0-gravity and 1-gravity) may have a strong impact on training of human gait. Gravity balancing exoskeletons are also invaluable in characterizing the short-term and long-term effects of the absence of gravity on human musculature, an important issue for astronauts and future manned programs in space. For a heavy manufacturing assembly line, an upper arm exoskeleton can be designed for an operator using the methods presented in this paper, with the specific requirements of the assembly task.

In the last two decades, robotics research has led to a variety of actively controlled machines, including designs of quadrupeds and bipeds that have provided a better understanding of balance during ambulation ([1], [2], [3], [4]). These machines use elaborate sensing, computation, and control to achieve their goals of navigation and manipulation. In recent years, a new use of robots is emerging for training of functional movements and gait in human ([5], [6], [7], [8], [9]). The robots act as both sensors and actuators for the human movement. However, with these machines, safety is of utmost importance and is of a concern to the clinicians. The unique feature of the gravity balancing exoskeleton proposed in this paper is its passivity, or the absence of actuators, which makes it inherently safe.

Gravity balancing has been used to reduce the actuator effort in machines during motion, through the clever use of counter-weights [10] and springs ([11], [12]) that make the system potential energy constant. A primary limitation of these proposed design procedures with springs is that the system loses its gravity balancing property if it changes its orientation with respect to the gravity vector [13], an issue of importance since the trunk continuously changes its orientation with respect to the gravity direction during walking. Gravity balancing has also been demonstrated by counterbalancing at the system center of mass [14]. or by inertially fixing it in space [15]. The exoskeleton designs presented in this paper first locate the center of mass of the system using auxiliary parallelograms and then springs are added through the center of mass and other locations such that the total potential energy of the system is invariant with configuration. This procedure 


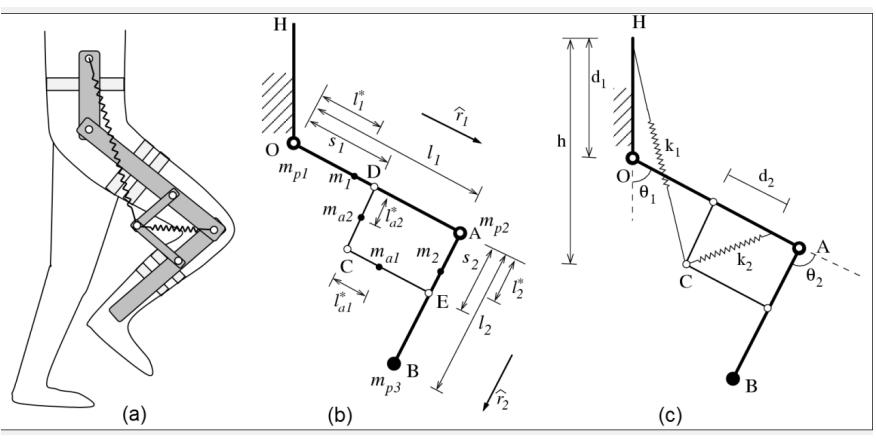

Fig. 1. (i) Basic components of a gravity balancing exoskeleton, (ii)Various terms and parameters of gravity balancing exoskeleton.

ensures gravity balancing even when the orientation of the system is changed with respect to the gravity vector [13]. This procedure was also applied to a spatial mechanism [16], where the joint axes are not parallel to each other. A unique feature of these gravity balanced designs is that they do not require actuators at the joints to keep the system balanced in every configuration.

\section{Design of A GRAVity BALANCing Exoskeleton}

The gravity balancing exoskeleton design consists of the following steps: (i) the center of mass of the leg is located using a parallelogram mechanism. (ii) one spring is connected through the center of mass and the other springs are placed at suitable locations so that the potential energy of the combined leg/exoskeleton system becomes invariant with configuration.

We consider the thigh and shank segments of the leg as distributed masses and the foot as a point mass in the design. A sketch of the leg with exoskeleton is shown in Fig. 1(a) and detailed geometric and inertial parameters of the human leg and exoskeleton are shown in Fig. 1(b) and (c). The segments $\mathrm{OA}$ and $\mathrm{AB}$ are the primary links of the exoskeleton, whereas $\mathrm{DC}$ and $\mathrm{CE}$ are the auxiliary links. The inertial parameters are as follows: $m_{i}$ is the mass of the $i^{t h}$ primary link that includes the mass of the human leg segment, $m_{a i}$ is the mass of the $i^{t h}$ auxiliary link, and $m_{p i}$ is the $i^{\text {th }}$ point mass. Here, $m_{p 3}$ includes the weight of the foot.

The geometric parameters are: $l_{i}$ is the length of the $i^{\text {th }}$ link, $l_{i}^{*}$ is the distance of the center of mass of the $i^{t h}$ primary link from the joint on the previous link, $l_{a i}^{*}$ is the distance of the center of mass of the $i^{t h}$ auxiliary link from the joint on the previous link, $s_{1}$ and $s_{2}$ are the distances OD and AE shown in Fig. 1(b). The vectors are defined as follows: $\widehat{\mathbf{r}}_{i}$ is the unit vector along the $i^{t h}$ primary link, $\mathbf{r}_{i}$ is the position vector from the point $\mathrm{O}$ to the center of mass of $i^{t h}$ primary link, $\mathbf{r}_{a i}$ is the position vector from the point $\mathrm{O}$ to the center of mass of $i^{t h}$ auxiliary link, and $\mathbf{r}_{p i}$ is the position vector from the point $\mathrm{O}$ to the center of mass of $i^{\text {th }}$ point mass.

Among all these quantities, only $m_{a i}, s_{i}$ and $l_{i}^{*}$ are unknown variables. Also, if we assume that the auxiliary links are made of telescopic members, their mass remains constant, independent of their length. $l_{a i}^{*}$ would become a linear function of the length of $i^{t h}$ auxiliary link. Hence, the only remaining unknown quantities are $s_{i}, i=1,2$. We define $l_{1}^{*}=\alpha_{1} l_{1}$, $l_{2}^{*}=\alpha_{2} l_{2}, l_{a 1}^{*}=\beta_{1}\left(l_{1}-s_{1}\right), l_{a 2}^{*}=\beta_{2} s_{2}, \alpha_{i}$ and $\beta_{i}$ are ratios between 0 and 1 .

The center of mass of the combined leg and exoskeleton is given by

$$
\mathbf{r}_{O C}=\frac{\sum m_{i} \mathbf{r}_{i}}{\sum m_{i}}
$$

where

$$
\begin{aligned}
\sum m_{i} \mathbf{r}_{i}= & m_{1} \mathbf{r}_{1}+m_{2} \mathbf{r}_{2}+m_{a 1} \mathbf{r}_{a 1}+m_{a 2} \mathbf{r}_{a 2}+ \\
& m_{p 1} \mathbf{r}_{p 1}+m_{p 2} \mathbf{r}_{p 2}+m_{p 3} \mathbf{r}_{p 3}, \\
\sum m_{i}= & m_{1}+m_{2}+m_{a 1}+m_{a 2}+m_{p 1}+ \\
& m_{p 2}+m_{p 3} .
\end{aligned}
$$

Since $\mathrm{C}$ is the center of mass of the entire mechanism, $\mathbf{r}_{O C}=s_{1} \widehat{\mathbf{r}}_{1}+s_{2} \widehat{\mathbf{r}}_{2}$. On substituting for $\mathbf{r}_{i}, \mathbf{r}_{a i}, \mathbf{r}_{p i}, l_{i}^{*}, l_{a i}^{*}$ and $\mathbf{r}_{O C}$ into Eq. (1) and solving for $s_{1}$ and $s_{2}$, we get [17]

$$
\begin{aligned}
s_{1} & =\frac{l_{1}\left(m_{1} \alpha_{1}+m_{2}+m_{p 3}+m_{a 1} \beta_{1}+m_{p 2}\right)}{m_{1}+m_{2}+m_{p 1}+m_{p 2}+m_{p 3}+m_{a 1} \beta_{1}} \\
s_{2} & =\frac{l_{2}\left(m_{2} \alpha_{2}+m_{p 3}\right)}{m_{1}+m_{2}+m_{a 2}+m_{p 1}+m_{p 2}+m_{p 3}-m_{a 2} \beta_{2}}(4)
\end{aligned}
$$

With the values of $s_{1}$ and $s_{2}$ given by Eq. (4), the center of mass of the whole mechanism including the human leg gets located in all configurations. It is important to point out that $s_{1}$ and $s_{2}$ are proportional to the lengths of primary links $l_{1}$ and $l_{2}$ and also depend on the mass distribution.

In the next step, the gravity balancing is achieved using springs located on the mechanism as shown in Fig. 1(c). Our designs use zero free-length springs, i.e., the rest lengths of the springs are zero [18]. Let $x_{1}$ and $x_{2}$ be the extended lengths of the springs with stiffness $k_{1}$ and $k_{2}$, respectively. Both springs have their one end attached at the center of mass C. For the gravity to be compensated completely, the total potential energy needs to be constant in all configurations. The expression for the total potential energy is given by $V=\frac{1}{2} k_{1} x_{1}^{2}+\frac{1}{2} k_{2} x_{2}^{2}+M g h$. Using geometry, once the expressions for $x_{1}^{2}, x_{2}^{2}$ and $h$ are substituted [17], we get

$$
V=C_{0}+C_{1} \cos \theta_{1}+C_{2} \cos \theta_{2}+C_{3} \cos \left(\theta_{1}-\theta_{2}\right),
$$

where

$$
\begin{aligned}
C_{0} & =\frac{1}{2} k_{1} d_{1}^{2}+\frac{1}{2} k_{2} d_{2}^{2}+\frac{1}{2} k_{1} s_{1}^{2}+\frac{1}{2} k_{1} s_{2}^{2}+\frac{1}{2} k_{2} s_{2}^{2}-M g d_{1} \\
C_{1} & =k_{1} s_{1} d_{1}-M g s_{1}, \quad C_{2}=k_{1} s_{1} s_{2}-k_{2} d_{2} s_{2} \\
C_{3} & =k_{1} s_{2} d_{1}-M g s_{2} .
\end{aligned}
$$

Note that all $C_{i}$ are constants, while $\theta_{i}$ represent the joint configuration of the leg. If the coefficients of terms containing $\cos \theta_{i}$ vanish, i.e., $C_{1}=C_{2}=C_{3}=0$, then the total potential energy is given by $V=C_{0}$, which is a constant. Thus, the total potential energy becomes configuration invariant and the gravity balancing is achieved. These conditions yield two independent equations:

$$
k_{1}=\frac{M g}{d_{1}}, \quad k_{2}=\frac{M g s_{1}}{d_{1} d_{2}} .
$$




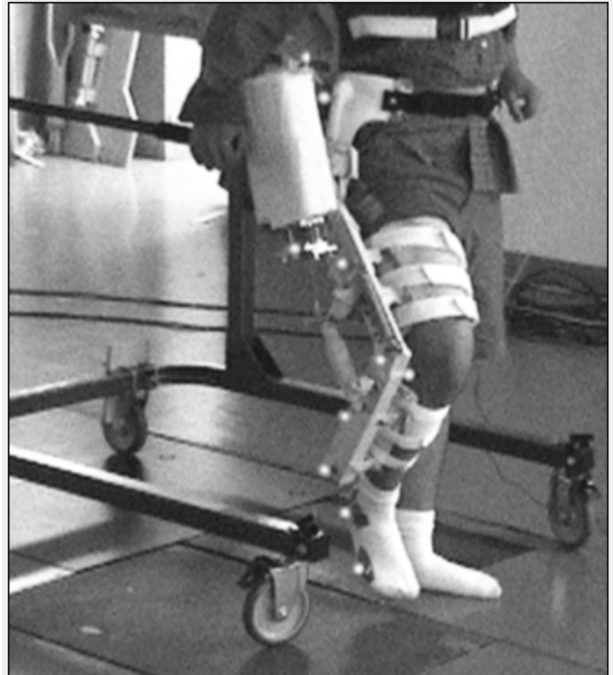

Fig. 2. Engineering prototype mounted on a walking frame and the subject in the gravity balancing device. To view videos, please see the footnote 3 .

Hence, if two zero free length springs with stiffness given by Eqs. (7) are used, the mechanism would become gravity balanced. Eq. (6) shows that the first spring $k_{1}$ compensates for the gravity force $M g$ of the total system and $k_{2}$ helps to make the potential energy invariant with configuration. $d_{1}$ and $d_{2}$ are arbitrary variables and can be chosen to vary the level of gravity balancing at the joints.

An engineering prototype was fabricated with the following features : (i) Limbs of the machine were made out of lightweight aluminum and are telescopic to accommodate variability in the leg dimensions and inertia across human subjects; (ii) The spring locations are adjustable to change the level of gravity during motion, between 0 - and 1-gravity [13]; (iii) The machine is supported on a walker frame and has attachments for the trunk and the limbs to conform to the contours of a human subject; (iv) Additional degreesof-freedom are added that allow the trunk to rotate about a vertical axis, pelvis to translate, and the hip to abduct and adduct; (vii) Joint encoders are mounted at the joints of the exoskeleton; and (viii) Two force-torque sensors are added at the interface between the human and machine limbs, on the thigh and the shank. The joint torques are computed through a free-body analysis of the human and machine limbs.

\section{DATA COLLECTION With THE EXoskelETON}

Experiments were conducted to evaluate the performance of the exoskeleton on human subjects by comparing leg muscle electromyography (EMG) recordings, leg joint range of motion measured using optical encoders, and leg joint torques measured using interface force-torque sensors. Two sets of experiments are described in this paper. Subjects gave informed consent according to procedures approved by the institutional review board of the University of Delaware.
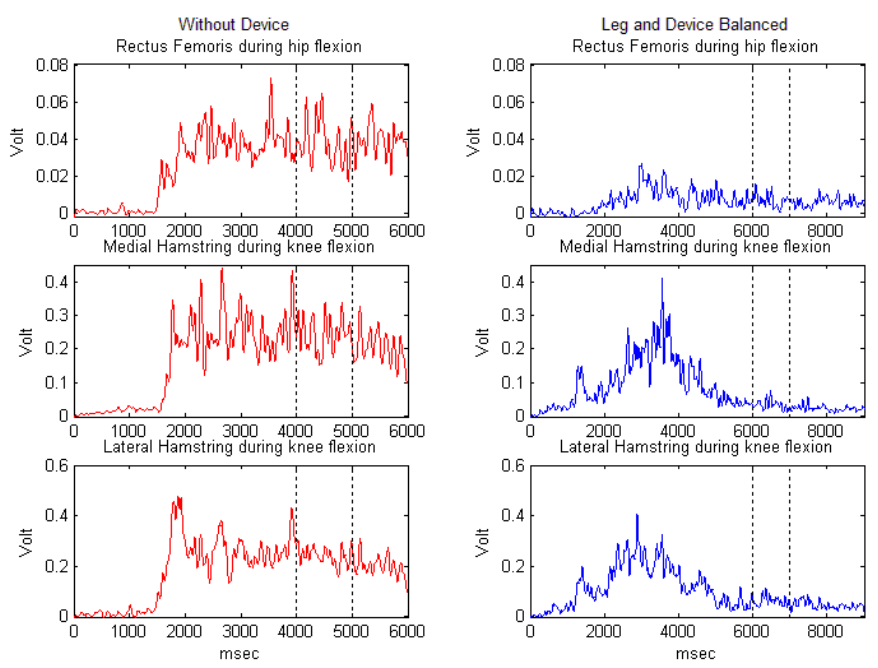

Fig. 3. Rectified and filtered EMG for a representative subject for the three muscles during the dynamic and static phases in two conditions, "without the device" and with "leg and device balancing".

\section{A. Experiment I: Tests in Static Configurations}

Five healthy young adults participated in this experiment. The subjects donned the device that was adjusted such that the hip and knee axes of rotation on the device were aligned with the corresponding axes of the subjects joints. The spring attachments of the device were adjusted to gravity balance the limb and the device, so that the subjects could position their limb in various configurations with their muscles relaxed. Subjects were required to perform two tasks. 1) Hip flexion: from $40 \mathrm{deg}( \pm 5 \mathrm{deg}$ standard error $(\mathrm{SE}))$ to $60 \mathrm{deg}(6 \mathrm{deg} \mathrm{SE}$ across subjects). 2) Knee flexion: from $65 \mathrm{deg}( \pm 6 \mathrm{deg} \mathrm{SE})$ to 72 deg ( \pm 7 deg SE). The knee angle in the hip flexion task and the hip angle in the knee flexion task were approximately the same. Subjects performed the static positioning experiments under two conditions: with the leg and device balanced and without device (trial duration, $9 \mathrm{sec}$ and $6 \mathrm{sec}$ respectively). Five trials were collected for each condition.

Figure 3 shows the rectified and filtered EMG for a representative subject for three muscles during the dynamic and static phases of the static positioning task involving either hip flexion or knee flexion, both with and without the device. The top panel shows rectus femoris EMG activity in the hip flexion task. The middle and bottom panels show the medial and lateral hamstring EMG activity during the knee flexion task. Note the lower activity of these muscles in the leg and device balanced condition (left panels) compared to the without device conditions. In the final resting position, the limb is expected to be gravity balanced. This can be seen in the $1 \mathrm{sec}$ interval indicated in Figure 3 between two dotted lines. We integrated EMG (IEMG) over a one second interval (corresponding to the dotted lines in Figure 3), when the limb was held static in the final, flexed position. IEMG for each muscle from the appropriate task (hip flexion for rectus femoris and knee flexion for the medial and lateral hamstrings) 


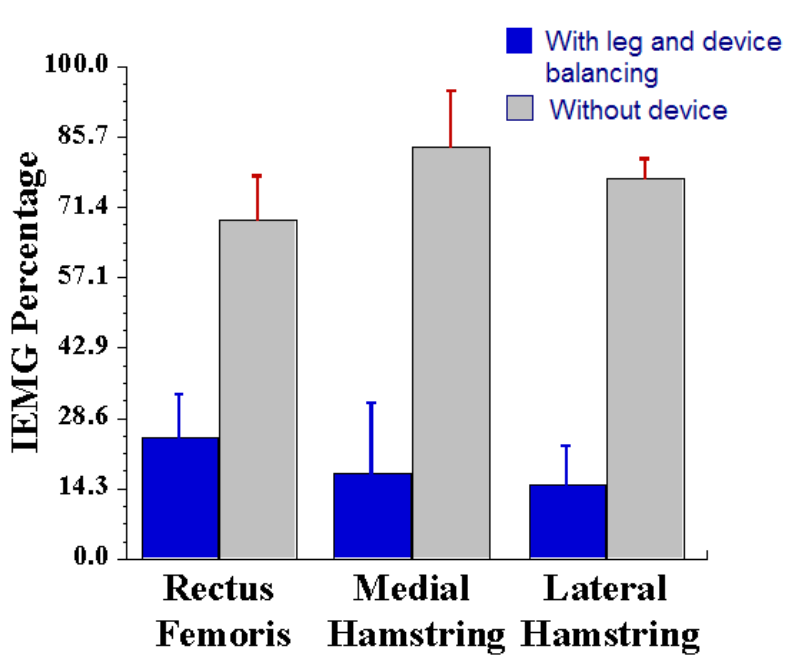

Fig. 4. IEMG percentages averaged across the five subjects for stepping task, with error bars.

in a subject was normalized to the maximum IEMG obtained from the five trials in the without device condition in that subject and expressed as a percentage. Figure 4 shows these IEMG percentages averaged across the five subjects with error bars. Note that the IEMG percentages for the leg and device balanced condition were always lower than for the without device condition $(p<0.05)$.

\section{B. Experiment II: Tests during Treadmill Walking}

For this experiment, five healthy subjects and an individual who had right hemiparesis following a stroke 2.5 years earlier walked on a treadmill. Five trials of $30 \mathrm{sec}$ duration were collected. Walking tasks were conducted within the device, with either both the leg and device gravity-balanced (leg and device balanced condition) or only the device gravity balanced (device only balanced condition) to compare the effects of gravity alone. EMG data of same muscles as above was collected along with joint motion data using optical encoders at the hip and knee joints. Joint torque data was recorded using two force-torque sensors mounted at the interface of human leg and device, one between the thigh segment of the device and the thigh of the subject, second between the shank segment of the device and the shank of the subject.

The healthy subjects walked at several different speeds on a treadmill while wearing the device under both conditions. The individual with right hemiparesis walked at his preferred walking speed of 1 mile/hour or $0.447 \mathrm{~m} / \mathrm{s}$. Therefore, the results for the healthy subjects presented here are for walking at the same approximate speed, which corresponded to $60 \%$ of their preferred speed. Very promising results were obtained from these experiments, in terms of increase in the joint range of motion, when using the exoskeleton. Fig. 5a shows the plots of the hip joint angle versus the knee joint angle of a representative healthy subject performing the walking task. It is clear from the plots that for the leg and device balanced condition, the range of movement at both hip and knee is larger
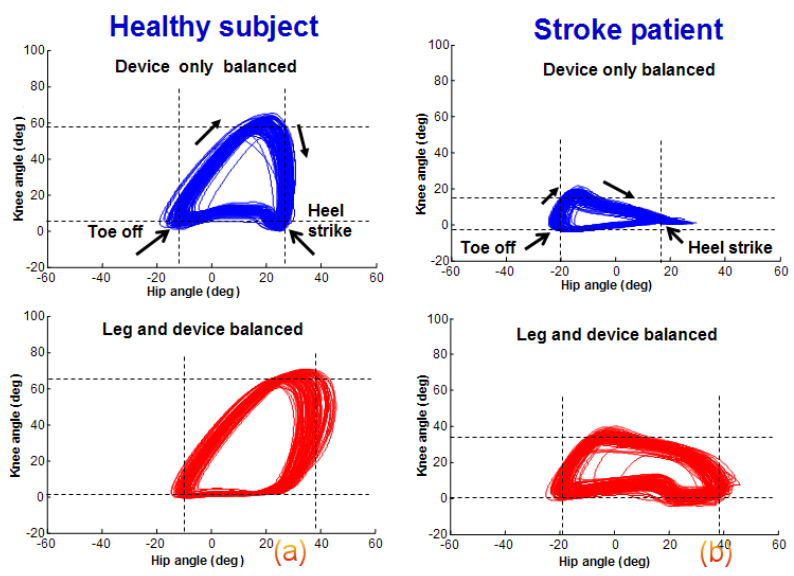

Fig. 5. Hip joint angle vs knee joint angle plot of a healthy subject and a stroke patient from walking tasks.
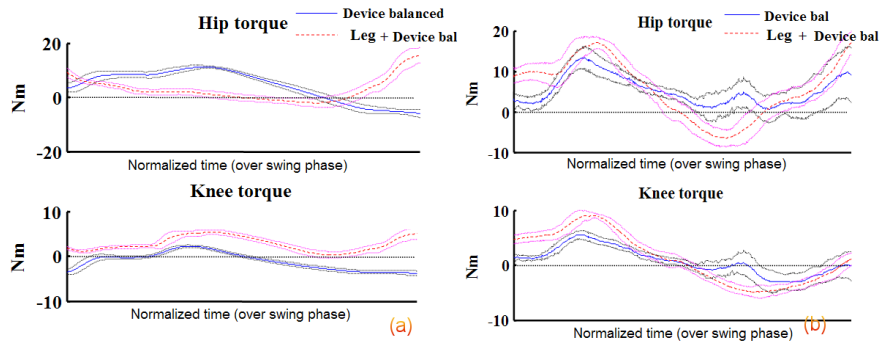

Fig. 6. Normalized torque (and its standard deviation) for walking task averaged over all trials of a representative subject and a stroke patient.

than with device only balancing. At hip joint, the increase in range was about $22 \%$ and at knee joint the increase in range is about $24 \%$. For the individual with a stroke, this increase in range of joint angles was more prominent than healthy subjects (Fig. 5b). The increase in range of joint angles was $45 \%$ at hip joint and $85 \%$ at knee joint. Furthermore, estimation of the step length showed an average increase in step length of $5.73 \%$ in the patient. This is an important positive effect of gravity balancing.

Figs. 6(a) and (b) show normalized torques for swing phase of walking averaged over all trials of one healthy subject and the individual with hemiparesis in the leg and device balanced and device balanced conditions. In the healthy subject, torque at hip joint is smaller for leg and device balanced condition compared with device balanced condition, for most of the swing phase. However, the knee joint doesnt show this reduction in torque. In the stroke patient, the joint torques showed no difference between conditions. Further analysis confirms that at the speed at which subjects are walking, inertial torque plays a significant role. Hence, gravity-balancing alone is likely inadequate to reduce the torque magnitudes. In addition, the passive elasticity of the muscles across human joints and friction in the joints of the machine were not accounted for. The patterns of muscle activation in individuals with stroke are known to be different from healthy subjects and may 
contribute to the lack of an effect of the device on joint torques. In walking tasks, the EMGs also did not show differences between the leg and device balanced and device balanced conditions. Despite the lack of effects related to EMG and torque for the stroke patient, the increase in range of motion of the joints that resulted from gravity-balancing of the leg and device has important implications for improvement in the patients gait pattern.

\section{CONCLUSIONS}

This paper presented the design principles and a prototype for a gravity balancing exoskeleton for the human leg. This exoskeleton is fully passive, i.e., does not use any actuators but still takes away the gravity load from the joints. It is adjustable to a subject wearing it. The exoskeleton was tested on five healthy human subjects and a patient with right hemiparesis following a stroke. The evaluation of this exoskeleton was perfomed by comparison of leg muscle EMG recordings, joint range of motion using optical encoders, and joint torques measured using interface force-torque sensors. The results showed that the average maximum EMG value for the "leg and device balanced" condition was around $25 \%$ of the EMG value for the "without device" conditions for the static experiments. In the walking experiments, there was a significant increase in the range of motion at the hip and knee joints for the healthy subjects and the stroke patient. For the stroke patient, the range increased by $45 \%$ at hip joint and by $85 \%$ at the knee joint. We believe that lower or upper extremity exoskeletons, that unload the human joints from gravity, can significantly enhance the human understanding of the role that gravity plays in human movement and can provide new insights into movement training.

\section{ACKNOWLEDGMENT}

The support of NIH grant \# 1 RO1 HD38582-01A2 is greatfully acknowledged.

\section{REFERENCES}

[1] S. C. Jacobsen, M. Olivier, F. M. Smith, D. F. Knutti, R. T. Johnson, G. E. Colvin, and W. B. Scroggin, "Research robots for applications in artificial intelligence, teleoperation and entertainment," International Journal of Robotics Research, vol. 23, no. 4-5, pp. 319-330, 2004.

[2] J. Schmiedler and K. J. Waldron, "Mechanics of quadrupedal galloping and the future of legged vehicles," International Journal of Robotics Research, vol. 18, no. 12, pp. 1224-1234, 1999.

[3] K. Loffler, M. Gienger, and F. Pfeiffer, "Sensors and control concept of walking johnnie," International Journal of Robotics Research, vol. 22, no. 3-4, pp. 229-239, 2003.

[4] P. Neuhaus and H. Kazerooni, "Industrial-strength human-assisted walking robots," IEEE Robotics and Automation Magazine, vol. 8, no. 4, pp. $18-25,2001$.

[5] R. A. Scheidt, D. J. Reinkensmeyer, M. A. Conditt, W. Z. Rymer, and F. A. Mussa-Ivaldi, "Persistence of motor adaptation during constrained, multi-joint, arm movements," J. Neurophysiol., vol. 84, pp. 853-862, 2000.

[6] T. Rahman, R. Ramanathan, S. Stroud, W. Sample, R. Seliktar, W. Harwin, M. Alexander, and M. Scavina, "Mtowards the control of a powered orthosis for people with muscular dystrophy," Proceedings of the Institution of Mechanical Engineers, Part H: Journal of Engineering in Medicine, vol. 215, no. 3, pp. 267-274, 2001.
[7] H. I. Krebs, J. J. Palazzolo, L. Dipietro, M. Ferraro, J. Krol, K. Rannekleiv, B. T. Volpe, and N., "Rehabilitation robotics: Performance-based progressive robot-assisted therapy," Autonomous Robots, vol. 15, no. 1, pp. 7-20, 2003.

[8] R. F. Macko, E. Haeuber, M. Shaughnessy, K. L. Coleman, D. A. Boone, and G. V. Smith, "Microprocessor-based ambulatory activity monitoring in hemiparetic stroke patients: Reliability and validity," Med Sci Sports Exerc, 2002.

[9] V. R. Edgerton, N. J. Tilakaratne, A. J. Bigbee, R. D. de Leon, and R. R. Roy, "Plasticity of the spinal circuitry after injury," Ann. Rev. Neurosci., vol. 27, pp. 145-167, 2004.

[10] V. H. Arakelin and M. R. Smith, "Complete shaking force and shaking moment balancing of linkages," Mechanisms and Machine Theory, vol. 34, pp. 1141-1153, 1999.

[11] L. F. Cardoso, S. Tomazio, and J. L. Herder, "Conceptual design of a passive arm orthosis," in In Proceedings, ASME Design Engineering Technical Conferences, 2002.

[12] T. Laliberte and C. Gosselin, "Static balancing of 3 dof planar parallel mechanisms," IEEE/ASME Transactions on Mechatronics, vol. 4, no. 4 , pp. 363-377, 1999.

[13] S. K. Agrawal and A. Fattah, "Theory and design of an orthotic device for full or partial gravity-balancing of a human leg during motion," IEEE Transactions on Neural systems and Rehabilitation Engineering, vol. 12 , no. 2, pp. 157-165, 2004.

[14] S. K. Agrawal, G. Gardner, and S. Pledgie, "Design and fabrication of a gravity balanced planar mechanism using auxiliary parallelograms," Journal of Mechanical Design, Transactions of ASME, vol. 123, no. 4 pp. 525-528, 2001.

[15] S. K. Agrawal and A. Fattah, "Reactionless space and ground robots: Novel designs and concept studies," Mechanisms and Machine Theory, vol. 39 , pp. 25-40, 2004.

[16] _ "Gravity-balancing of spatial robotic manipulators," Mechanisms and Machine Theory, vol. 39, pp. 1331-1334, 2004

[17] S. K. Banala, S. K. Agrawal, A. Fattah, K. Rudolph, and J. Scholz, "Gravity balancing leg orthosis for robotic rehabilitation," in the IEEE Proceedings on International Conference of Robotics and Automation, 2004, pp. 2474-2479.

[18] D. A. Street and B. J. Gilmore, "Perfect spring equilibrators for rotatable bodies," ASME Journal of Mechanisms, Transmissions, and Automation in Design, vol. 111, no. 4, pp. 451-458, 1989. 